\title{
Myrmedobia und Lichenobia,
}

zwei neue einheimische Rhynchoten-Gattungen.

Von

Prof. Dr. v. Baerensprung.

\section{面yrmedobia.}

Nachdem Westwood $\left.{ }^{1}\right)$ die Gattung Microphysa mit einer Art M. pselaphiformis (pselaphoides Burm.) aufgestellt und ihre verwandtschaftlichen Beziehungen zu Anthocoris und Xylocoris erkannt hatte, beschrieb Märkel ${ }^{2}$ ) eine zweite Art, die in Gesellschaft von Ameisen lebt und von ihm M. Myrmecobia genannt wurde. Ger mar ${ }^{3}$ ) liefs sie bald nachher sehr naturgetreu abbilden. Diese Art war aber nicht neu, sondern schon von Fallén ${ }^{4}$ ) als Salda coleoptrata beschrieben. Sie mufs wegen der abweichenden Bildung der Oberflügel und der Schnabelscheide von Microphysa getrennt werden und eine eigene Gattung bilden, für die ich den Namen Myrmedobia vorschlage. Zu dieser Gattung gehört noch eine zweite, bisher unbeschriebene Art, welche sich in der hiesigen Königlichen Sammlung befindet.

Die Gattung Myrmedobia theilt mit Microphysa die Kleinheit und Form des Körpers im Allgemeinen; namentlich den dreieckigen, vorn in eine stumpfe Spitze verlängerten Kopf, das kurze Brustschild und den breiten, fast kreisrunden Hinterleib; aber bei Myrmedobia ist die ganze Oberfläche stärker gewölbt und glänzend. Der Kopf ist mehr nach abwärts geneigt, dicker; neben den ziem-

1) Annales d. l. soc. entomol. de France Vol. III.

$\left.{ }^{2}\right)$ Zeitschrift für die Entomologie von Germar Vol. V.

$\left.{ }^{3}\right)$ Fauna Insector. Europae fasc. XXIV.

4) Monograph. Cimic. Sueciae.

Berl. Entomol. Zeitschr. I. 
lich stark hervortretenden Netzaugen bemerkt man jederseits ein kleines Grübchen, welches zur Aufuahme eines Nebenauges bestimmt zu sein scheint, ohne dafs ein solches darin zu erkennen ist. Die Fühler sind bei beiden Gatlungen ziemlich gleich gebildet, von etwas mehr als halber Körperlänge, kurz behaart, viergliedrig; alle Glieder cylindrisch. Dabei sind mit Ausnahme des ersten, welches kürzer und etwas dicker ist, die drei folgenden Glieder von ziemlich gleicher Länge; bei Microphysa auch von gleicher Stärke, während bei Myrmedobia die gröfsere Schlankheit des mittleren auffällt. Die Schnabelscheide ist bei Mierophysa wie bei den verwandten Gattungen dünn und reicht bis an das Ende der Brust: das erste Glied ist sehr kurz, das zweite bei Weitem das längste, das dritte und vierte haben gleiche Länge. Myrmedobia dagegen hat eine auffallend dicke Schnabelscheide, welche kaum über das erste Fufspaar hinausreicht, und deren zweites Glied nur wenig länger als das vorhergehende und als die folgenden ist; alle Glieder sind dick und etwas plattgedrückt, das letzte kegelförmig zugespitzt.

Der Thorax ist bei Microphysa trapezförmig, nach vorn verschmälert, sein hinterer Rand breit, ausgeschweift mit spitz hervortretenden Hinterecken; seine Oberfläche zeigt eine von zwei Querfurchen begrenzte Wulst. Bei Myrmedobia ist er fast rechteckig, doppelt so breit als lang, die Vorderecken sind abgerundet, seine Oberfläche ist glatt oder quer eingedrückt.

Die Beine sind in beiden Gattungen schlank, ohne Auszeichnung; die Tarsen zweigliedrig, das letzte Glied mit zwei kleinen Krallen.

Die Oberflügel haben bei Microphysa eine Beschaffenheit, welche auch sonst in der Ordnung der Rhynchoten, namentlich in den Familien der Capsinen, Lygaeoden und Reduvinen häufig vorkommt; sie sind lederartig, bedecken den Hinterleib nur zur Hälfte, und sind hinten schräg abgestutzt. Es ist augenscheinlich, dafs zu ihrer vollständigen Entwickelung etwas fehlt, und dafs der hintere abgestutzte Rand eigentlich dazu bestimmt ist, eine Membran zu tragen. Diese Vermuthung wird dadurch noch unterstützt, dafs man am Grunde des Koriums zwei nach hinten divergirende, aber bald verlöschende Nerven bemerkt, welche ein Randfeld, ein Mittelfeld und einen Klavus, freilich nur andeutungsweise, abgrenzen. Ich bin also der Meinung, dafs man die Oberflügel bei Microphysa als unvollständig entwickelte Halbdecken mit fehlender Membran zu betrachten habe, und entnehme eine Bestätigung dafür besonders aus der Vergleichung mit dem bekannten Xylocoris rufipennis L. I) uf. (Lyctoco. 
ris corticalis $\mathrm{Hahn.).} \mathrm{Während} \mathrm{nämlich} \mathrm{bei} \mathrm{allen} \mathrm{übrigen} \mathrm{Arten}$ der Gattung Xylocoris die Oberflügel aus Korium, Klavus, Kuneus und Membran zusammengeselzt sind, findet man bei der genannten Art eine Flügelbildung, die im Wesentlichen ganz mit der bei Microphysa übereinstimmt. Myrmedobia dagegen hat wirkliche Flügeldecken, wie sie den Käfern zukommen, aber nur bei wenigen Rhynchoten gefunden werden: Flügeldecken von harter, horniger Beschaffenheit, welche den Hinterleib vollständig bedecken und keine Andeutung einer Nervenvertheilung bemerken lassen. Unterflügel fehlen bei beiden Gattungen.

Das Schildchen ist mäfsig grofs, dreieckig, unbedeckt.

Der Hinterleib breit, kurz und dick, fast sphärisch.

Die Gattungs - Charaktere von Myrmedobia lassen sich hiernach folgendermafsen zusammenstellen:

Corpus parvum, rotundatum, convexum, nitidum. Caput triquetrum, oculis prominentibus, stemmatis nullis. Rostrum crassum et breve, articulis quatuor cylindricis, subaequilongis, ultimo conico. Antennae 4-articulatae, articulo primo crassiore, sequentibus cylindricis, subaequilongis, tertio ceteris paullum tenuiori. Pronotum transversum, subparallelogrammum, angulis anticis rotundatis. $P e-$ des graciles, tarsis 2-articulatis. Scutellum triangulare. Elytra convexa, abdomen globosum prorsus tegentia, cornea, enervia. Alae nullae.

Die beiden mir bis jetzt bekannten Arten der so charakterisirten Gattung sind:

1. Mr. coleoptrata: fusco-nigra, nitida, subtilissime aureo-pubescens, vertice, rostro abdomineque supra rufis, antennis basi pedibusque pallidis, thorace laevi. - Long. $\frac{2}{3}$ lin.

Salda coleoptrata Fallén. Mon. Cimic. Suec. „Species omnino distincta, huic generi (Salda) affinis, minuta, magnitudine quasi Sinapis dimidia. Corpus ferrugineum, capite rufo. Elytra valde convexa, nigra, nitida, abdomen tegentia, tomento brevi aureo obsita; membrana nulla. Alae nullae. Pedes et antennae pallida. Larva imagini similis, ferruginea, elytris destituta."

Microphysa Myrmecobia Märkel Zeitschrift für die Entomolog. Vol. V. „Fusco-nigra, nitida, pubescens, vertice, rostro abdomineque rufis, coleopteris subhemisphaericis, convexis, punctatis, nigris, antennis basi pedibusque pallidis. Variat capite toto rufo. Specimina elytris abbreviatis, apice oblique truncatis, ocellis carentia larvas credo."

Germar. Faun. Insect. Furopae. fasc. XXIV. tab. 19. 
Eine der kleinsten Wanzen, wenig über eine halbe Linie lang. Die Oberfläche des Körpers stark gewölbt, sehr fein goldgelb behaart, seidenartig glänzend. Brustschild, Schildchen und die ganze Unterseite des Körpers pechbraun, die Flügeldecken glänzend schwarz, der Kopf rostroth, vorn dunkler, hinten heller, in der Mitte ein dunkler Punkt. Die beiden ersten Fühlerglieder und die Beine blafsgelblich. Durch die starke Wölbung des Kopfes sind der Grund des Schnabels und die Augen etwas nach unten gerückt; der mittlere, schmale Lappen des Kopfschildes tritt vorn als eine kleine Spitze hervor. Der kurze, dicke Schnabel ist in der Ruhe knieförmig gegen die Brust aufwärts gebogen; sein Grund von dem breit lanzettförmigen Labrum bedeckt. Die Fühler sind unmittelbar vor und unter den Augen eingelenkt, schlank, ziemlich dicht und kurz behaart; das erste Glied das kürzeste, die drei folgenden von ziemlich gleicher Länge, das dritte merklich dünner als das vorhergehende und das folgende. Brustschild oben glatt, ohne Eindruck, doppelt so breit als lang, die Hinterecken rechtwinklig, die Vorderecken abgerundet. Schildchen vorn etwas eingedrückt. Beine kurz behaart. Die fein punktirten Flügeldecken liegen dicht aneinander und sind stark gewölbt, so dafs sie den Hinterleib vollständig bedecken; sie haben ihre gröfste Breite etwas hinter der Mitte. Der Hinterleib ist oben rostroth, unten braun, fast kuglig; bei einigen Individuen, die ich für Männchen halte, etwas schmäler als bei den andern. Andere Geschlechtsunterschiede sind nicht zu bemerken. Neben den vollständig ausgebildeten Exemplaren kommen andere vor, welche statt der Flügeldecken nur kurze Flügelstumpfe haben. Da sie in allen übrigen Beziehungen mit jenen völlig übereinstimmen, so ist es zweifelhaft, ob man sie nit Fallén und Germar für Larven halten darf.

Fallén, welcher diese Art in Schweden entdeckte, bemerkt, dafs sie im Sande am Ufer von Seen vorkomme und auch von Gyllenhal im Grase gefunden worden sei. Schiödte fand sie in Dänemark und Märkel in Sachsen in den Nestern der Formica rufa. Bei Berlin wurde sie von Herrn Tieffenbach in einer hohlen Eiche, gleichfalls in Gesellschaft von Ameisen, beobachtet. In der Sammlung des Professor Kirschbaum sah ich ein unausgefärbtes, blafsbraunes Exemplar, welches von ihm in der Nähe von Wiesbaden, und ein zweites ausgefärbtes, welches von Förster bei Aachen gefunden worden war. 
2. W1. Mfoscutellata: fusco-nigra, nitida, subtilissime punctata, vertice, rostro, scutello elytrorumque margine summo rufis; pedibus pallidis, thorace sulco transverso impresso. - Long. $1 \frac{1}{4}$ lin.

Diese Art hat im Habitus viele Aehnlichkeit mit Microphysa pselaphoides, aber durch die angegebenen Gattungscharaktere schliefst sie sich der vorigen Art nahe an, von der sie sich aufser durch die ansehnlichere Gröfse durch folgende Eigenthümlichkeiten unterscheidet. Der ganze Körper ist etwas weniger gewölbt, stärker punktirt, nicht seidenartig behaart; der Kopf etwas schmaler, länger, die Augen seitlich stärker hervortretend; die Schnabelscheide verhältnifsmäfsig weniger dick und das zweite Glied derselben verhältnifsmäfsig etwas länger als bei M. coleoptrata. Der Thorax hat dieselbe rechteckige Form mit abgerundeten Vorderecken, ist aber oben nicht glatt, sondern in die Quere tief eingedrückt. Die Flügeldekken sind ebenfalls stark konvex und bedecken den ganzen Hinterleib; aber sie sind am Grunde mehr erweitert, so dafs ihre gröfste Breite nicht hinter, sondern in der Mitte liegt; ihr Aufsenrand ist nach oben umgebogen; Brust und Bauch sind wie bei der vorigen* Art pechbraun; die Flügeldecken glänzend schwarz, Kopf und Schnabelscheide roth, aber aufserdem haben auch noch das Schildchen und der umgebogene Rand der Flügeldecken dieselbe rostrothe Färbung. Der Grund der Fühler ist braun; die Beine sind blafsgelblich.

Ein einziges Exemplar findet sich in der hiesigen Königlichen Sammlung mit folgender Bezeichnung: ,Kellberg; an faulen Schwämmen; sehr seltene Wanze." Es stammt von Waltl in Passau.

\section{Hichenobia.}

An die Microphysa - und Myrmedobia-Arten schliefst sich eine andere, ebenfalls sehr kleine und bisher unbeschriebene Wanze an, welche als Repräsentant einer eigenen Gattung betrachtet werden mufs. Die Verwandtschaft mit Myrmedobia spricht sich zunächst in dem Mangel der Unterflügel und in der Beschaffenheit der Oberflügel aus, welche auch hier wahre Flügeldecken sind und den Hinterleib vollkommen bedecken.

Der Kopf hat dieselbe dreieckige und gewölbte Form; die seitlich ziemlich stark hervortretenden Netzaugen und keine Nebenaugen. Der Schnabel ist wie bei Microphysa dünn, und reicht bis ans Ende der Brust; er ist viergliedrig; das erste Glied kurz, dick und von dem schmal lanzettförmigen Labrum bedeckt; das zweite wenig länger als das erste; das dritte Glied länger und dünner; das 
vierte das längste und dünnste, so lang als die vorhergehenden zusammengenommen.

Die 4gliedrigen Fühler haben eine Beschaffenheit, welche von der bei Microphysa und Myrmedobia abweicht, und unter den verwandten Gattungen nur bei Xylocoris vorkommt; die beiden ersten Glieder sind cylindrisch, am Eude etwas verdickt; die beiden letzten dagegen ganz fein, borstenförmig und ziemlich lang behaart.

Der Thorax ist an seiner Oberfläche glatt, ohne Eindruck und von fast quadratischer Form, nämlich an seinem Hinterrande kaum etwas breiter als in der Mitte lang, mit fast parallelen Seitenrändern aber abgerundeten Vorderecken.

Die Beine sind von mäfsiger Länge; die Koxen des vorderen Paares einander sehr nahe gerückt und etwas verlängert; die Schenkel und Schienen desselben Paares gegen das Ende hin etwas verdickt. Die Tarsen sind zweigliedrig, zweiklauig. Das zweite Fufspaar ist das kürzeste, das dritte das längste.

Das Schildchen ist mäfsig grofs, dreieckig, unbedeckt. Der Hinterleib ist von den Flügeldecken ganz bedeckt, hat hier aber nicht eine breite, sphärische Form, sondern ist schmal, walzenförmig, hinten kaum etwas breiter als an seinem Grunde. Dadurch wird nun auch die Form des ganzen Körpers eine länglich gestreckte.

Eigentliche Flügel fehlen, wie bei den zunächst verwandten Gattungen, und die Oberflügel sind wahre Flügeldecken von schmaler, aber gewölbter, der Gestalt des Hinterleibes entsprechender Form, welche nicht übereinander greifen, sondern nebeneinander liegen und nicht aus mehreren Stücken zusammengesetzt sind, sondern aus einer einzigen Hornplatle bestehen. Was sie aber von den Flügeldecken bei Myrmedobia wesentlich unterscheidet, ist, dafs sie von deutlichen Nerven durchzogen sind. Von dem Grunde jeder Flügeldecke aus verlaufen zwei Nerven bis zu ihrem hintern Ende, der eine dem Aufsenrande, der andere dem Innenrande einigermafsen parallel. Ersterer schickt einen queren Ast zum Aufsenrande, und endlich sind beide Längsnerven noch vor ihrem Ende durch einen zweiten queren Ast miteinander verbunden. Hierdurch zerfällt das ganze Korium in fünf Felder, nämlich in ein langes und schmales Mittelfeld; in ein hiervon nach innen gelegenes, ebenfalls langes und schmales, den ganzen Innenrand der Flügeldecke begleitendes und in drei kleinere, am Aufsenrande und Hinterrande gelegene Felder oder Zellen.

Die Gattungscharaktere von Lichenobia sind hiernach:

Corpus parvum, oblongum, convexum. Caput triquetrum oculis 
prominentibus, stemmatis nullis. Rostrum longum et tenue, articulo primo brevissimo, secundo paullulum longiore, quarto longissimo. Antennae 4-articulatae, articulis primo breviore et secundo longiore cylindricis, tertio et quarto subaequilongis, tenuissimis, setaceis. Pronolum laeve subquadralum, angulis anticis rolundatis. Pedes anteriores femoribus et tibiis modice tumidis; pedes intermedii et posteriores graciles; tarsis 2-articulatis. Scutellum triangulare, liberum. Elytra convexa, cornea, diaphana, areolata, abdomen cylindricum prorsus tegentia. Alae nullae.

Die einzige mir bis jetzt bekannte Art ist:

L. Fenpergimea: fusco-ferruginea, rostro, elytris pedibusque pallidis, pilosis, abdomine supra coccineo. - Long. 1 lin.

Körper ziemlich schmal eiförmig, hinten wenig breiter als vorn, mäfsig gewölbt, glatt, mattglänzend, braun, Schnabelscheide, Fühler und Beine blasser, Rücken des Hinterleibes mit Ausnahme der Spitze roth. Kopf gleichseitig dreieckig, vorn abgerundet, gewimpert, oben glatt. Keine Nebenaugen. Schnabel hinter den beiden ersten, kürzeren und dickeren Gliedern knieförmig gebogen, lang und dünn. Thorax fast so lang als breit, glatt; neben den abgerundeten Vorderecken jederseits ein kleiner Eindruck. Fühler ziemlich lang behaart, von halber Körperlänge; das erste Glied kurz, das zweite doppelt so lang: beide cylindrisch, gegen das Ende hin etwas verdickt; das dritte und vierte Glied untereinander gleich lang, länger als das zweite, sehr fein, borstenförmig. Vorderbeine ziemlich lang und stark; besonders sind die Schenkel und Schienbeine gegen das Ende hin etwas verdickt und borstig behaart; das mittlere und hintere Fufspaar ist schlanker, die Hinterschienen etwas verlängert. Tarsen 2gliedrig mit 2 kleinen Krallen. Die Flügeldecken haben eine hornartige Transparenz und lassen die oben beschriebene Aederung erkennen. Eigentliche Flügel fehlen. Das Schildchen ziemlich spitz dreieckig; der Hinterleib walzenförmig, oben abgeflacht, hinten spitz zulaufend. Das letzte ziemlich lange Segment ist unten ausgeschnitten für die Mündung des Afters und der Geschlechtstheile. Beim Männchen ist der Hinterleib etwas kürzer, beim Weibchen etwas länger als die Flügeldecken, und zugleich breiter und gewölbter.

Herr Wagenschieber entdeckte diese Art in einer Anzahl von Exemplaren bei Berlin in den Nestern der Formica rufa. Ein einzelnes Exemplar wurde von Herrn Habelmann auf der Insel Wollin unter Moos gefangen. Die beiden von mir untersuchten Exemplare, ein Männchen und ein Weibchen, befinden sich in der Sammlung des Herrn Stein. 
Zur Vergleichung lasse ich schliefslich noch die Gattungscharaktere von Microphysa folgen:

\section{Microphysa Vestw.}

Corpus parvum, triquetrum, modice convexum, opacum. Caput triquetrum, stemmatis nullis. Rostrum tenue, articulo primo brevissimo, secundo ceteris longiore, tertio et quarto subaequilongis. Antennae 4-articulatae, articulo primo crassiore, sequentibus cylindricis, subaequilongis. Pronolum trapezoideum, antice angustatum, margine postico arcualo, angulis posticis acutis, supra transverse rugosum. Pedes graciles, tarsis 2-articulatis. Scutellum triangulare, liberum. Hemelytra coriacea, abbreviata, oblique truncata, abdomen globosum dimidio tegentia. Alae nullae.

Mredopteysa pselaphoiates Burm.: Fusco-grisea, vertice rufo; antennarum articulo secundo, geniculis, tibiis et tarsis pallidis, abdomine nigro. - Long. $1 \frac{1}{4}$ lin.

Von Curtis in England, von L. Dufour in Frankreich, von Boheman in Schweden, von Sahlberg in Finnland, von Erichson in der Berliner Gegend entdeckt, scheint die Microphysa ein weit verbreitetes Vaterland zu haben. Sie ist bei Berlin nicht selten unter der Rinde von Ahorn, Platanen und Weiden; besonders häufig fand ich sie aber im Berliner Thiergarten an einem alten Bretterzaun in Gesellschaft der Formica quadripunctata während der Monate Juli und August. Die etwas kleineren und schmaleren Männchen sind seltener als die Weibchen. Die Larven, welche neben den erwachsenen Thieren häufig angetroffen werden, unterscheiden sich von den letzteren durch den Mangel der Oberflügel und den viel schmaleren Thorax, welcher ebenso wie der Kopf, die Fühler und Beine ganz hellroth ist, während der sphärische Hinterleib sich durch seine glänzend schwarze Färbung auszeichnet. Die Fühler sind bei den Larven wie bei den ausgewachsenen Exemplaren viergliedrig.

(Die zu diesem Aufsatze gehörigen Abbildungen folgen im nächsten Jahrgang.) 


\section{$2 \mathrm{BHL}$ Biodiversity Heritage Library}

1857. "Myrmedobia und Lichenobia, zwei neue einheimische Rhynchoten $\square$ Gattungen.'Berliner entomologische Zeitschrift / herausgegeben von dem Entomologischen Vereine in Berlin 1(1), 161-168.

https://doi.org/10.1002/mmnd.18570010117.

View This Item Online: https://www.biodiversitylibrary.org/item/34385

DOI: https://doi.org/10.1002/mmnd.18570010117

Permalink: https://www.biodiversitylibrary.org/partpdf/210170

\section{Holding Institution}

Smithsonian Libraries

\section{Sponsored by}

Smithsonian

\section{Copyright \& Reuse}

Copyright Status: Public domain. The BHL considers that this work is no longer under copyright protection.

This document was created from content at the Biodiversity Heritage Library, the world's largest open access digital library for biodiversity literature and archives. Visit BHL at https://www.biodiversitylibrary.org. 